patients; $77 \%$ had residual arterial abnormalities, with improvement in $45 \%$ and stabilization in $32 \%$. Before the arteriopathy stabilized or improved, transient worsening occurred in 14 $(19 \%)$ of TCA patients; $13(18 \%)$ had a recurrent stroke or TIA. Stroke was preceded by chickenpox in $44 \%$ of TCA patients and in none of those with progressive arteriopathies. Neurological outcome was good in $30(41 \%)$ of the TCA patients and in none of the 5 with progressive arteriopathy. Progressive arteriopathy was associated with arterial occlusion, moyamoya disease and anterior cerebral artery involvement, and with stroke recurrence. (Braun KPJ, Bulder MMM, Chabrier S, et al. The course and outcome of unilateral intracranial arteriopathy in 79 children with ischemic stroke. Brain Feb 2009;132:544-557). (Respond: KPJ Braun MD, PhD, Department of Child Neurology, Rudolf Magnus Institute of Neuroscience, Wilhelmina Children's Hospital, University Medical Center Utrecht, PO Box 85500, 3508 GA Utrecht, the Netherlands. E-mail: k.braun@umcutrecht.nl).

COMMENT. In contrast to adults with stroke, the majority of childhood arterial ischemic strokes are caused by non-atherosclerotic arterial disease. Transient cerebral arteriopathy is a common cause and is characterized by infarction in the lateral lenticulostriate territory. The majority of patients in the above study had unilateral transient cerebral arteriopathy, and stroke was preceded by chickenpox (post-varicella arteriopathy) in $44 \%$. Evidence supports a post-infectious inflammatory mechanism underlying transient cerebral arteriopathy.

\title{
CLASSIFICATION OF PERINATAL ISCHEMIC STROKE
}

Advances in classification, causes, treatment and outcome of perinatal ischemic stroke are reviewed by researchers at Alberta Children's Hospital, Calgary; and the Hospital for Sick Children, Toronto, Canada. Four perinatal stroke syndromes are described in order of decreasing incidence: 1) neonatal arterial ischemic stroke (accounts for two-thirds); 2) neonatal cerebral sinovenous thrombosis; 3) presumed perinatal ischemic stroke; and 4) periventricular venous infarction. MRI of the head and neck is the investigation of choice in perinatal stroke. Diffusion-weighted MRI provides information on timing for acute, focal infarction. MR angiography defines arterial occlusion or arteriopathy. MR venography defines location and extent of venous thrombosis, and diffusion weighted MRI confirms or excludes venous edema or infarction.

Maternal, prenatal, and placental risk factors include chorioamnionitis, prolonged rupture of membranes, preeclampsia, placental thrombi and intrauterine growth retardation. Intrapartum factors include neonatal encephalopathy with asphyxia that may co-occur with perinatal stroke. Prothrombotic and hematological factors occur in $20-68 \%$ of neonates with ischemic stroke. Cardiac evaluation is recommended in all cases of perinatal stroke, and complex congenital heart disease predisposes neonates to cerebral thromboembolism, especially during diagnostic and surgical procedures. Infection and inflammation are common in perinatal stroke, and the association with bacterial meningitis and chorioamnionitis requires further study.

Treatment involves maternal prevention approaches (counseling regarding smoking, weight control), rescue at risk brain tissue (anticoagulation, maintain normal blood sugar, temperature, oxygenation, blood pressure), and optimize outcome (study of hypothermia, treatment of seizures). Congenital hemiplegia and epilepsy are the most common 
neurological deficits, and disorders of language, vision and cognition occur in $20-60 \%$ of perinatal stroke cases. Neuroimaging lesion size and location are helpful in prediction of outcome. Basal ganglia involvement correlates with poor prognosis. (Kirton A, deVeber G. Advances in perinatal ischemic stroke. Pediatr Neurol March 2009;40:205-214). (Respond: Dr Kirton, Division of Neurology, Alberta Children's Hospital, 2888 Shaganappi Trail NW, Calgary AB T3B 6A8, Canada. E-mail: adam.kirton@calgaryhealthregion.ca).

COMMENT. The distinction between specific causative factors and coincidental associations is a challenge, particularly with prenatal factors in the etiology of perinatal ischemic stroke. Advances in neuroimaging (functional MRI, diffusion tensor imaging, and transcranial magnetic stimulation) have improved prediction of outcome and should increase understanding of brain reorganization and plasticity.

Risk of epilepsy after perinatal stroke was studied by retrospective review of 64 children followed after 6 months of age at Indiana University School of Medicine (Garg BP et al. J Pediatr 2007;151:409-413; Ped Neur Briefs Oct 2007;21:79). Neonatal seizures were recorded in the NICU in $75 \%$ of cases. Epilepsy had developed in $67 \%$ between age 6 months and follow-up at a mean age of 43 months. Infarct on prenatal ultrasound and family history of seizures were significantly associated with development of epilepsy following perinatal stroke.

\section{TRAUMATIC DISORDERS}

\section{SPINAL SUBDURAL HEMATOMA AND NON-ACCIDENTAL HEAD INJURY}

The incidence of spinal pathology in 18 children with non-accidental head injury was determined in a study at Nottingham University Hospitals, UK. Between 2000 and 2007 children with non-accidental head injury had MRI of brain and whole spine; the spine was examined routinely in all suspected cases after 2005. Spinal subdural hematoma occurred in $8(44 \%)$ cases. It was clinically occult in all cases, usually thoracic or lumbar in location, and large in 6 . All had supratentorial and infratentorial intracranial subdural hematomas. Three of the 8 patients with supratentorial subdurals had skull fractures. Follow-up MRI of the spine within 1 to 3 months in 6 of the 8 cases showed reduction in size of the spinal subdural in 5 and complete resolution in 1. (Koumellis P, McConachie NS, Jaspan T. Spinal subdural hematomas in children with non-accidental head injury. Arch Dis Child March 2009;94:216219). (Respond: Dr T Jaspan, Radiology Department, B Floor, Queen's Medical Centre, Derby Road, Nottingham NG7 2UH, UK. E-mail: tim.jaspan@nuh.nhs.uk).

COMMENT. A high incidence of occult spinal subdural hematoma is reported in children with non-accidental head injury and brain subdurals. Patients with suspected nonaccidental head injury should receive MRI of brain and whole spine in all cases. 